\section{Arc minute gravitational lenses and cosmic strings}

PACZYŃSKI has suggested ${ }^{1}$ that several large separation gravitational lens candidates could be explained by the existence of cosmic strings. These lens candidates are quasar pairs with separation between 1 and 4 arc min. Paczyński states that arc minute separation quasar pairs, which are lensed by long straight strings, are a prediction of cosmic string theory. Paczyński's work received a great deal of attention when preliminary evidence for a gravitational lens with a 2.6 arc min separation was published by Turner et $a l^{2}$. Because a lens with such a large separation is difficult to explain with unseen conventional objects and requires a large mass-tolight ratio for the lens, this lens candidate has been reported ${ }^{3}$ as evidence for a cosmic string. Subsequent observations suggest that the quasar pair is not lensed ${ }^{4}$. We point out here that such large separation lenses are not predicted by cosmic string theories.

The typical separation of the images of an object that is lensed by a cosmic string $^{5-7}$ is $4 \pi G \mu$ where $G \mu$ is the dimensionless mass parameter of the string. A gravitational lens with a separation of $2.6 \mathrm{arcmin}$ corresponds to $G_{\mu} \approx$ $6 \times 10^{-5}$. While it is possible for a string configuration to produce gravitational lenses with image separations $>4 \pi G \mu$ with favourable geometry, to increase the image separation by more than a factor of two or three above this value seems to require very unlikely conditions. While it was originally suggested ${ }^{8-10}$ that a valve of $G \mu \simeq 10^{-3}-10^{-5}$ might be useful for galaxy formation, further work has shown that these valves are too high, and probably already excluded by observation. Arc minute separation lenses are thus not a prediction of cosmic string theories. If some of these objects were lensed by long straight strings, it would create other cosmological problems, or suggest a basic misunderstanding of how cosmic strings should behave.

We now list the evidence against valves of $G \mu>10^{-5}$. Models where galaxies or clusters accrete around loops ${ }^{11,12}$ have obtained $G \mu=2 \times 10^{-6}$. Thus the prediction of cosmic string models is that $G \mu \simeq$ $10^{-6}$ not $10^{-5}$. More serious problems for large values of $G \mu$ come from nucleosynthesis and anisotropy of the microwave background radiation. The most stringent limit on $G \mu$ comes from the requirement that the strings do produce so much gravitational radiation as to interfere with the successful primordial nucleosynthesis scenario ${ }^{13-15}$. This bound is stringent, $G \mu<4 \times 10^{-6}$, but it can also be avoided by some types of cosmic strings that predominantly radiate massless goldstone

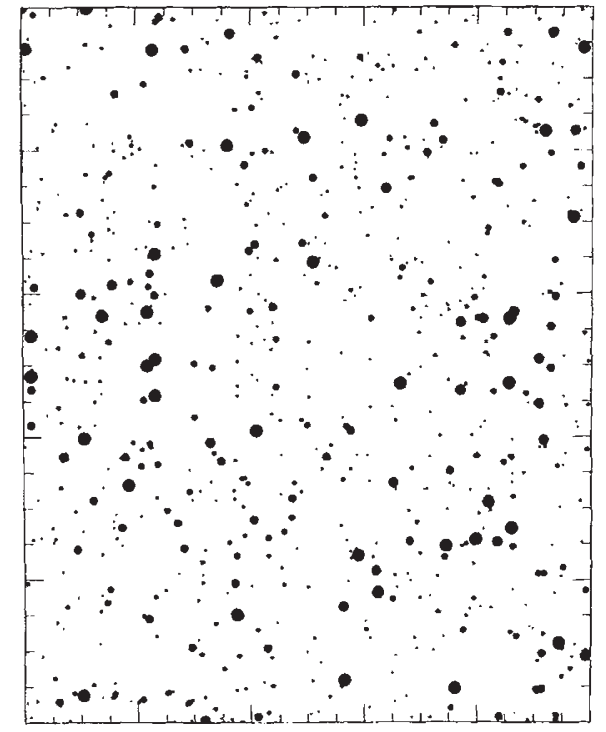

Fig. 1 Computer-generated image of 700 stars in a flat three-dimensional space, with a broad luminosity function.

bosons rather than gravitational waves ${ }^{16}$. However, these strings are not as attractive as seeds for galaxy formation, nor do they appear in any attractive particle physics models. A completely model-independent limit has been obtained by Kaiser and Stebbins ${ }^{17}$ who showed that $G \mu<10^{-5}$ is required by the observed isotropy of the microwave background. Similar limits come from anisotropy due to string loops and the gravitational waves they produce $^{18,19}$. While estimating the errors on these theoretical limits is difficult, we feel that at least the limit of Kaiser and Stebbins is actually conservative. As they stated, the limit given is for a "minimal model" which, for example, does not include the increase in the r.m.s. temperature fluctuation due to the superposition of strings which will occur in any model. Thus, the limit $G \mu<10^{-5}$ is quite firm, so the predicted separation of images lensed by a cosmic string is definitely $<1$ arc min.

This work was supported by the Department of Energy, contract DE AC03 $76 \mathrm{SF} 00515$.

MS 209,

DAVID P. BENNETT

Fermilab,

P.O. Box, 500, Batavia,

Illinois 60510, USA

Physics Department Albert STEBbins

University of California, Berkeley, California 94720, USA

1. Paczyńskí, P. Nature 319, 567-568 (1986)

2. Turner, E. L. et al. Nature 321, 142-144 (1986)

3. Gott, J. R. Nature 321, 420-421 (1986)

4. Shaver, P. A. \& Christiani, S. Nature 321, 585-586 (1986).
5. Vilenkin, A. Astrophys. J. 282, L51-53 (1984)

6. Gott, J. R. Astrophys. J. 288, 422-427 (1985)

7. Frieman, J. in Inner Space Outer Space (eds Kolb, E. W., Turner, M. S., Lindley, D., Olive, K. \& Seckel, D. 540-542 (University of Chicago Press, 1986)

8. Zel'dovich, Y. B. Mon. Not. R. Astr. Soc. 192, 663-667 (1980).

9. Vilenkin, A. \& Shafi, Q. Phys. Rev. Lett. 51, 1716-1719 (1983).

10. Turok, N. Nucl. Phys. B242, 520-541 (1984)

11. Turok, N. \& Brandenberger, R. Phys. Rev. D33, 2175-2181 (1986).

12. Stebbings, A. Astrophys. J. 303, L21-25 (1986)

13. Davis, R. L. Phys. Lett. 161B, 285-288 (1985).

14. Bennett, D. P. Phys. Rev. D33, 872-888 (1986) erratum (in the press)

15. Bennett, D. P. Phys. Rev. (in the press)

16. Davis, R. L. Phys. Rev. D32, 3172-3177 (1985).

17. Kaiser, N. \& Stebhins, A Nature 310, $39 t-393$ (1984)

18. Brandenberger, R. H. \& Turok, N. Phys. Rev. D33, 2182 2195 (1986).

19. Traschen, J., Turok, N. \& Brandenberger, R. Microwave Anisotropies from Cosmic Strings, ITP Preprint, NSF. ITP-85-110 (1985).

PACZYŃSKI REPLIES-In my letter ${ }^{1}$ I intended to emphasize two issues. Firstly, it is unlikely that cosmic strings could be discovered by finding pairs of images. I pointed out that there is a more striking effect of a string lensing: the images of some galaxies would appear as if they were cut with a sharp edge. Secondly, I pointed out that with the images separated by a few arc minutes the expected difference in the light travel time between the two images may be a few hundred or even a few thousand years. We do not know how stable are the broad line spectra of quasars, and I presented some observational reasons why they may, in fact, be variable. Therefore, I proposed that the forbidden lines in the spectra of pairs of quasars may help to decide between a cluster of quasars and a gravitational lens hypothesis. I quoted some papers (not mine) that suggested that strings may produce pairs of images separated by up to a few arc minutes, but this was just one of the many possibilities I listed. Therefore, it is surprising that Bennett and Stebbins give me so much credit for the cosmic string as the cause of the 2.6 arc min lens candidate.

I would like to take this opportunity to re-emphasize the same two points. Figure 1 shows a computer-generated field with about 700 'stars' randomly placed in a flat three-dimensional space, with a broad luminosity function. Half-way between us and the 'horizon' there is a straight 'cosmic string' that produced 27 pairs of images. Readers are encouraged to find the pairs and the string. This can be done, but the presence of the 'string' is not striking. That was my first point ${ }^{1}$.

I do not think that it is possible to prove or disprove the gravitational lens hypothesis for the 2.6 arc min candidate of Turner et al. by analysing their spectra, as the forbidden lines are too weak to be seen. Fortunately, the quasars (or the quasar) are relatively close, with redshift, $z=1.01$. With present technology it is 